\title{
Crystallographic Characterization of Black Phosphorene and its Application in Nanostructures
}

\author{
Yi Ren, Pu Liu, Benliang Zhou, Xiaoying Zhou $\oplus^{*}$ and Guanghui Zhou $\oplus^{\dagger}$ \\ Department of Physics and Key Laboratory for Low-Dimensional Quantum Structures and Manipulation (Ministry \\ of Education), and Synergetic Innovation Center for Quantum Effects and Applications of Hunan, \\ Hunan Normal University, Changsha 410081, China
}

(Received 27 August 2019; revised manuscript received 29 October 2019; published 10 December 2019)

\begin{abstract}
The central question in the field of two-dimensional (2D) materials is how a material behaves when it is patterned at nanometer scale with different edge geometries. Due to the anisotropy inherent in the puckered structure, black phosphorene nanostructures may have more varieties of edge geometries. Here, we present a comprehensive 2D planar crystallographic characterization of phosphorene uniformly by a chiral vector (angle), from which we report a type of edge atomic configuration, the slope-edge geometry. The phosphorene nanoribbons (PNRs) with slope edges, like previously noticed zigzag and skewed-armchair PNRs, also possess interesting twofold-degenerate edge states. These three marginal directions, together with the skewed-zigzag and armchair directions without edge states, divide a phosphorene into four regions, among which the electronic properties are different from each other. Further, for a PNR cutting along any possible direction, by taking into account the $z$ direction in the structure, whether or not it possesses edge states depends on the existence of periodic zigzaglike morphology along the edges. For application, moreover, we propose a PNR-based Z-shaped homogenous junction with scale approximately $100 \mathrm{~nm}$, where the central scattering region between two PNR electrodes is a phosphorene quantum dot (PQD) of a PNR segment with various edge morphologies. Interestingly, the calculated conductance by the KWANT code, based on tight-binding combined with a scattering matrix for the junction, relies sensitively on the central PQD edge states. In specification, for the junctions of PQDs with edge states, the conductance exhibits a terrace with resonant oscillations near the Fermi energy; otherwise, the electron transport is blocked due to the absence of edge states. Remarkably, the number of oscillating peaks exactly matches the number of sawtooth along an edge of the PQD, since the discrete energy levels of the zigzaglike edge provide the transporting channels for electrons. The results here may be extended to the group-VA 2D materials in order to observe the electronic properties of nanostructures in experiments and provide a reference for the preparation of better nanodevices.
\end{abstract}

DOI: 10.1103/PhysRevApplied.12.064025

\section{INTRODUCTION}

In the current decade, two-dimensional (2D) materials have emerged as one of the most exciting classes of materials since the rise of graphene [1]. Recently, semiconducting black phosphorous (or phosphorene), a monoor few-layer structure of phosphorus atoms forming 2D puckered sheets, has joined the class of $2 \mathrm{D}$ materials [2-7]. The synthesized phosphorene has a noticeable band gap and appreciable anisotropy in its physical properties

\footnotetext{
*xiaoyingzhou@hunnu.edu.cn

†'ghzhou@hunnu.edu.cn
}

Published by the American Physical Society under the terms of the Creative Commons Attribution 4.0 International license. Further distribution of this work must maintain attribution to the author(s) and the published article's title, journal citation, and DOI.
[7-9]. Generally, a 2D material may display properties that differ greatly from those of its bulk counterpart due to the effects of quantum confinement and the large surface-tovolume ratio. Further, tailoring a $2 \mathrm{D}$ material along its different crystallographic directions, one can obtain onedimensional (1D) nanoribbons with a width less than 100 $\mathrm{nm}[10-14]$. These nanoribbons show not only the confinement effect but also a peculiar edge effect, both of which play an important role in the electronic, optical, magnetic, and transport properties [15-18]. Hence the edge states are particularly important in a 2D material nanoribbon.

Black phosphorus, with an anisotropic structure, is a van der Waals bonded layered material in which each layer forms a puckered surface due to $s p^{3}$ hybridization and possesses a direct band gap of $0.3 \mathrm{eV}$ [19]. This direct gap increases up to approximately $2 \mathrm{eV}$ as its thickness decreases from bulk to a few layers [20]. In addition, the field-effect transistor (FET) $[2,5,6]$ based on phosphorene 
is found to have an on-off ratio of $10^{3}$ and a high hole-carrier mobility up to $800 \mathrm{~cm}^{2} / \mathrm{V} \mathrm{s}$ [21]. Importantly, there have been a few effective ways to solve the instability problem for phosphorene in the environment [22]. These good properties of phosphorene have attracted broad interest [23-27]. Further, PNRs made by tailoring a phosphorene sheet in the conventional zigzag and armchair directions have been prepared experimentally in Refs. [28] and [12], respectively. The zigzag PNRs (ZPNRs) with significant edge states and armchair PNRs (APNRs) with a direct band gap have been extensively studied [13,16,17,29]. Also, some of the properties of PNRs have been verified $[18,30-36]$, such as the effect of edge states on the conductance and the thermal power [37], the influence of vacancy defects on the electron structure and on transport [38,39], and the electric tunable edge-pseudospin valve [40].

On the other hand, nanostructures with various possible edge atomic configurations have become a hot topic since the rise of $2 \mathrm{D}$ materials. A central question in the field of graphene-related research is how it behaves as patterned at the nanoscale with different edge morphological geometries $[10,41]$. If we cut a graphene sheet along the crystal directions by increasing the chiral angle from $0^{\circ}$ to $30^{\circ}$, for example, the edge geometry switches from zigzag to armchair, between which we can obtain all possible chiral ribbons with different chiral angles [10,41]. Recently, the effects of chirality and edge geometry on the anisotropic thermal transport properties have also been studied for $\mathrm{MoS}_{2}$ nanoribbons [42]. For PNRs, in addition to the conventional zigzag and armchair edges, other edge geometries have been predicted. For example, PNRs with stable beard-zigzag [29], skewed-zigzag (SZ, so SZPNR), and skewed-armchair (SA, so SAPNR) $[14,43,44]$ edges have also been reported and some of them have already been realized experimentally [45]. Essentially, the electronic properties are significantly different from each other among PNRs with different chiralities. This difference results in an abundant electrical nature, such as metals with edge states and semiconductors with a direct band gap. Furthermore, the difference in physical properties has potential applications. For instance, the control of external electric and magnetic fields on the electronic structure [46] and the coupling and manipulation of the edge states in multilayer PNRs [47]. However, the existing studies have only involved PNRs with the few particular abovementioned edge geometries. A uniform verification of the edge morphology for highly anisotropic phosphorene, such as those for isotropic graphene [10,41], is also important in phosphorene-based nanotechnology.

In this work, we first present theoretically the 2D planar crystallographic characterization of an anisotropic phosphorene. We define a chiral number (vector) or chiral (azimuthal) angle to uniformly describe the planar crystallographic directions. As the chiral angle increases from $0^{\circ}$ to $90^{\circ}$, as shown in Fig. 1, the boundary morphology

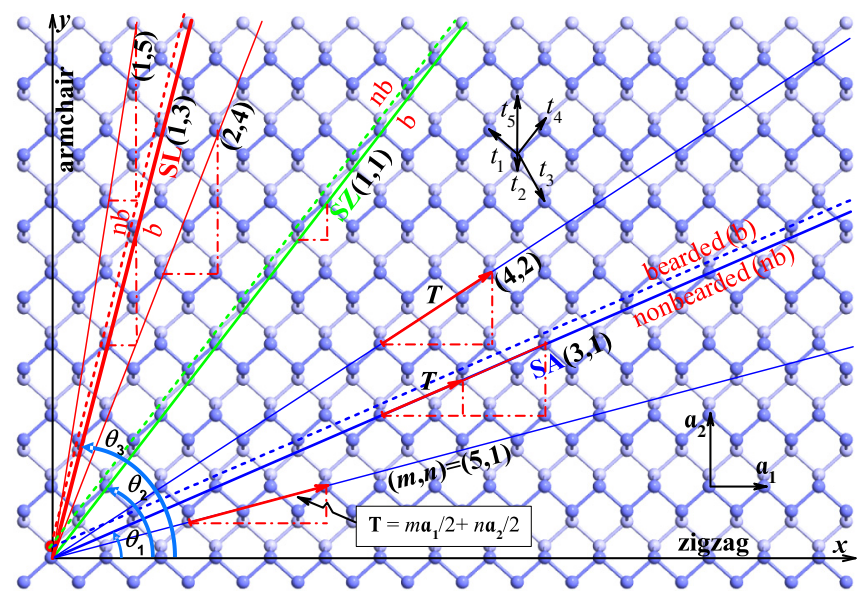

FIG. 1. An illustration of crystallographic characterization on a monolayer phosphorene, where the heavy (light) gray spheres donate the phosphorus atoms in the upper (lower) subplane and the $x$ and $y$ coordinates are along the zigzag and armchair crystallographic directions, respectively. The thick blue, green, and red solid lines are along the SA, SZ, and SL directions, with chiral angles $\theta_{1}, \theta_{2}$, and $\theta_{3}$, respectively; $\mathbf{a}_{1}$ and $\mathbf{a}_{2}$ denote the primitive vectors; and $t_{1}-t_{5}$ are the five hopping parameters. The chiral vector $\mathbf{T}$ is illustrated by the thick red solid arrow along each $2 \mathrm{D}$ crystallographic direction, where the arrows along $(4,2)$ and $(3,1)$ show that examples of the outermost atoms are located at the same sublayer and, alternatively, at two sublayers, respectively.

switches from zigzag to armchair and sweeps over all possible edge geometries during the process. We find that a phosphorene can be divided into four fan-shaped regions determined by the chiral angles $0^{\circ}, \theta_{1}\left(23.74^{\circ}\right)$, $\theta_{2}\left(52.84^{\circ}\right), \theta_{3}\left(75.82^{\circ}\right)$, and $90^{\circ}$, among which the electronic characteristics are different. Therefore, by cutting a phosphorene along these five regional boundary directions, one can obtain the previously studied ZPNR, SAPNR, SZPNR, and APNR $[14,43,44]$. Importantly, the PNR with a sloped (SL) edge geometry along $\theta_{3}$ (termed a SLPNR) between the SZ and the armchair direction has not yet been reported. Essentially, the SL direction is similar to the SA, with a periodic zigzaglike edge morphology supporting edge states. Further, by taking into account the effect of the $z$ direction in the structure, we then demonstrate the variation of the electronic properties for PNRs with different edge atomic geometries by the KWANT code based on tight-binding (TB) calculations [48], in which particular attention is paid to the SLPNR, which intrinsically has twofold-degenerate edge states. In addition, the origin of the bearded and nonbearded edges along each regional boundary direction is discussed and related to the existence of edge states. The results here may be extended to the cousins of phosphorene, such as 2D group-VA arsenene, antimonene, and bismuthene [49].

Second, based on the 2D crystallographic characteri zation, we propose a two-terminal Z-shaped homogenous 
junction based on PNR and calculate its transport properties using the KWANT code based on TB [48] combined with a scattering-matrix approach from wavefunction matching [50-52] for treating large enough systems comparable with experimental samples. The two electrodes of the junction are semi-infinite ZPNRs with the same width of approximately $100 \mathrm{~nm}$, where the central scattering region of a segment of PNR with edge-tilting angles from $0^{\circ}$ to $90^{\circ}$ is sandwiched between them. In consequence, the two hypotenuse edges of the central phosphorene quantum dot (PQD) sweep over all possible geometrical morphologies. Interestingly, the conductance spectrum depends sensitively on the tilting angle of the Z-shaped junction. For the junctions of PQDs with edge states, the transport behavior exhibits a conductance terrace with resonant oscillation in the low-energy regime; otherwise, the electron transport is blocked due to the absence of edge states. Remarkably, the number of conductance oscillating peaks is exactly equal to the number of outermost phosphorus atoms in the zigzaglike edges of the center PQD, since the discrete energy levels of the zigzaglike atomic configurations provide the transport channels for the electrons. These findings may provide a reference for the experimental preparation of better nanodevices.

This paper is organized as follows. In Sec. II, we first present the 2D crystallographic characterization of phosphorene, in which the crystallographic direction on the plane is uniformly described by a chiral vector or angle. By this vector (angle), the crystallographic directions with possible boundary edge geometries are identified, particularly including the newly recovered slope edge. Further, the calculation method using the KWANT code based on TB combined with a scattering-matrix approach is described. In Sec. III, a few examples of typical PNRs with a stable structure are first analogized and then the PNR-based Z-shaped junction with different tilting degrees, in which the central PQD with various edge morphologies is constructed, and the transport properties are studied in detail. Also, the main results are presented numerically, together with the microscopic explanation and discussion. Finally, we summarize our results in Sec. IV.

\section{MODEL DESCRIPTION AND METHOD}

\section{A. 2D crystallographic characterization}

Phosphorene is a typical orthorhombic-structured 2D material with two principal crystallographic directions, the zigzag and armchair directions, as shown in Fig. 1 and denoted as the $x$ axis and $y$ axis, respectively. It is known that the period of minimum rotation angle for graphene is $30^{\circ}$ from the zigzag to the armchair direction [10,41]. Due to the highly anisotropic structure of phosphorene, however, it needs to rotate $90^{\circ}$ anticlockwise from the zigzag to the armchair direction. In consequence, more possible directions may exist along which PNRs form by cutting a phosphorene in addition to along the conventional zigzag and armchair directions, as well as the previously verified SA and SZ directions $[14,44]$. Therefore, a unified identification for the crystallographic direction on 2D phosphorene is still in great demand.

The low symmetry of phosphorene leads to higher structural complexity compared to other 2D materials $[10,41,42]$ such as graphene, silicene, hexagonal BN ( $h$ $\mathrm{BN}$ ), and $\mathrm{MoS}_{2}$, all of which have a regular hexagonal symmetry in 2D. In previous works, the symmetry of phosphorene has been studied in terms of point-group theory [53]. In order to simply characterize the crystallographic directions for phosphorene by using the inversion symmetry to reduce the degree of freedom [29,53], here we first ignore the $z$ direction in the structure and add its effect on nanostructures in the next step. This simplification means that there are only two atoms in a primitive cell instead of the standard four atoms per primitive cell. Therefore, the 2D planar crystallographic directions of a phosphorene can be characterized by an orientational (chiral) index $(m, n)$ or, equivalently, a chiral angle $\theta$. Further, $(m, n)$ or $\theta$ also determines a minimum unit vector along the corresponding crystallographic direction. Therefore, we can define a chiral vector to characterize the crystallographic directions, which is given by

$$
\mathbf{T}=m \mathbf{a}_{1} / 2+n \mathbf{a}_{2} / 2 .
$$

Here, $\mathbf{a}_{1}$ and $\mathbf{a}_{2}$ are the primitive vectors of phosphorene, with amplitudes $a_{1}=3.32 \AA$ and $a_{2}=4.38 \AA$ along the $x$ and $y$ directions [54], respectively. The module of $\mathbf{T}$ is the length of the hypotenuse of the red dot-dashed-line right-angled triangle for each direction, shown in Fig. 1. Hence $m$ and $n$ are integers for the projection of $\mathbf{T}$ onto the $x$ and $y$ directions in terms of $a_{1} / 2$ and $a_{2} / 2$, respectively. In order to ensure that both $m$ and $n$ are integers, the two sides of the right-angled triangle in Fig. 1 are taken as half of the primitive vectors. Thus, we can obtain a series of possible crystallographic directions of phosphorene only when $m+n=$ even, because the $m+n=$ odd case is unphysical according to our definition of $\mathbf{T}$. To satisfy the condition for the chiral index, both $m$ and $n$ must simultaneously be even or odd. As a result, their difference is also an even integer. In particular, $|m-n|=0$ corresponds to chiral index $(m, n)=(1,1)$ for the SZ direction. In addition, when the difference is a nonzero even number, the corresponding chiral indexes are $(3,1),(1,3)$, and $(5$, 1), and so on, respectively (also see Fig. 1).

Meanwhile, we can accordingly introduce a chiral angle as

$$
\theta=\arctan \left(n a_{2} / m a_{1}\right)
$$

which connects with the chiral index $(m, n)$. Therefore, a crystallographic direction on the phosphorene plane can be 


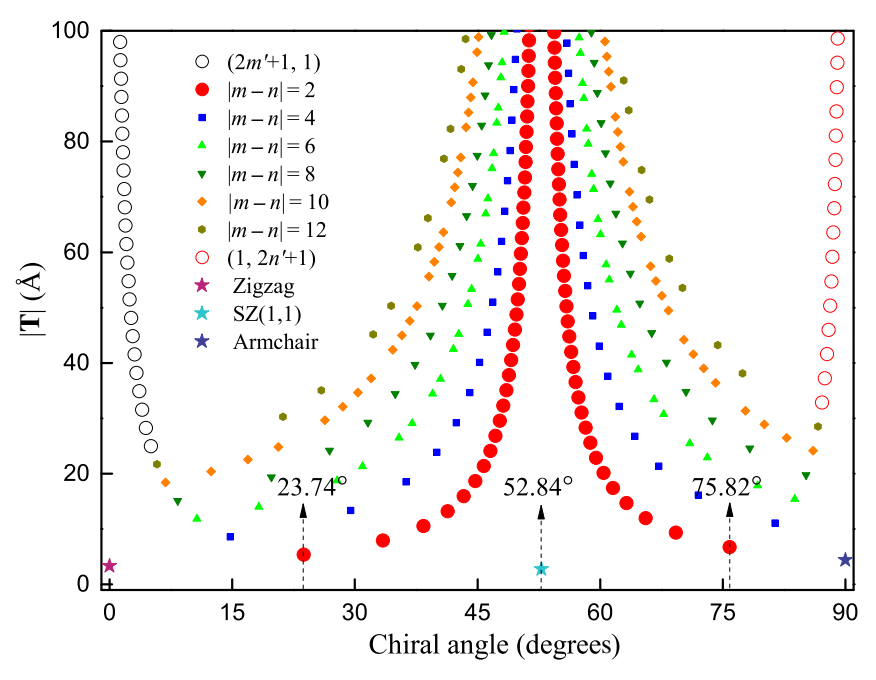

FIG. 2. The length of the unitary chiral vector $\mathbf{T}$ as a function of the chiral angle, where the lengths indicated by the red spheres plus the black and red circles form a distributional pattern with two separated $U$ shapes in which all the other dots are inside the valleys, except for the three colored stars corresponding to chiral angles $0,52.84$, and $90^{\circ}$, respectively.

characterized by either a chiral vector $\mathbf{T}$ or index $(m, n)$, or angle $\theta$. Despite all this, however, the structural characterization is still very different from that for graphene with a regular hexagonal symmetry [41].

For specification, in Fig. 2 we present $T$ (the modulus of $\mathbf{T}$ ) as a function of $\theta$ from $0^{\circ}$ (zigzag) to $90^{\circ}$ (armchair), where the different-colored and -shaped dots denote the different combinations of $m$ and $n$. For example, the red spheres indicate the value of $T$ for $|m-n|=2$, the blue squares for $|m-n|=4$, and so on. Importantly, the black and red circles denote the values for $\left(2 m^{\prime}+1,1\right)$ and $\left(1,2 n^{\prime}+1\right)$, which correspond to the directions with chiral angle near 0 and $90^{\circ}$, respectively. As shown in Fig. 2, the distribution of $T$ varying with $\theta$ for the directions simultaneously obeying $|m-n|=2,\left(2 m^{\prime}+1,1\right)$, and $\left(1,2 n^{\prime}+1\right)$ forms an interesting pattern of two separated $U$ shapes. All other points with different chiral indexes are located inside the $U$ shapes, except for the three special points indicated by stars. One is the split point indicated by a green star between two $U$ shapes at $\theta=52.84^{\circ}$ (SZ direction), along which $T=\left(a_{1}^{2}+a_{2}^{2}\right)^{1 / 2}$. The other two (brown and blue) star points at $\theta=0^{\circ}$ and $90^{\circ}$ correspond to the two boundaries of the zigzag and armchair, respectively. Further, the bottoms of the two $U$ shapes are at the two special chiral angles of $23.74^{\circ}$ and $75.82^{\circ}$, respectively, which correspond to the SA and the discovered SL directional boundaries. Moreover, with the increase of $|m-n|, T$ becomes longer and the atomic configuration of the corresponding boundary turns out to be more complicated.

Based on the above description, we conclude that, as shown in Fig. 1 and Table I, a phosphorene can essentially be divided into four major regions as the chiral vector rotates around the $z$ axis from the zigzag direction at chiral angle $\theta=0^{\circ}$ to the armchair at $90^{\circ}$. These four regions are also indicated by the angle ranges of $0-\theta_{1}\left(23.74^{\circ}\right), \theta_{1}-\theta_{2}\left(52.84^{\circ}\right), \theta_{2}-\theta_{3}\left(75.82^{\circ}\right)$, and $\theta_{3}-90^{\circ}$, with the regional boundaries determined by the five crystallographic directions along the zigzag, the SA (thick blue solid line), the SZ (thick green solid line), the SL (thick red solid line), and the armchair, respectively. More specifically, the crystallographic directions of the reginal boundaries are described by the indexes $(2,0),(3,1)$, $(1,1),(1,3)$, and $(0,2)$, respectively, at $\theta=0, \theta_{1}, \theta_{2}, \theta_{3}$, and $90^{\circ}$ or, equivalently, by the unitary-vector length $T=a_{1}$, $\left(9 a_{1}^{2}+a_{2}^{2}\right)^{1 / 2} / 2,\left(a_{1}^{2}+a_{2}^{2}\right)^{1 / 2} / 2,\left(a_{1}^{2}+9 a_{2}^{2}\right)^{1 / 2} / 2$, and $a_{2}$. We note that, with the exception of the discovered SL, the other four peculiar directions and their corresponding PNRs have been previously studied $[14,43,44]$. PNRs cutting along the SL direction and also with stable edges, however, have not yet been reported.

Further, other crystallographic directions also exist within each region, which are shown in an exemplary manner in Fig. 1 by the thin solid lines. Along these directions, the projection of unitary vector $\mathbf{T}$ on the horizontal (vertical) axis can be obtained by the combination of $a_{1} / 2\left(a_{2} / 2\right)$. For instance, when the chiral angle rotates anticlockwise from $\theta_{1}$ with $(m, n)=(3,1)$, the vertical component of $\mathbf{T}$ is always kept as $a_{2} / 2$, since the data are in the left periphery of the first "U" (see Fig. 2) and the horizontal one can be added with integer times of $a_{1}$, such as $(5,1)$, shown by the thin blue solid lines in Fig. 1. The subsequent possible crystallographic directions in this region are listed in the first column of Table I. Similarly, when the chiral angle rotates anticlockwise from $\theta_{1}$ to $\theta_{2}$ (SA to SZ), the corresponding horizontal (vertical) component of $\mathbf{T}$ varies in times of $a_{1} / 2\left(a_{2} / 2\right)$, such as $(4,2)$. A series of possible directions is also presented in the second column of Table I. However, according to the above derivation rule, we cannot obtain the varying law for boundaries from SZ to the armchair direction. Therefore, to complete the phase diagram, the discovered SL directional boundary must be filled in the region from the SZ to the armchair. When the chiral angle rotates clockwise from $\theta_{2}$ to $\theta_{3}$ (SL to SZ), the horizontal (vertical) projection of $\mathbf{T}$ varies with times of $a_{1} / 2\left(a_{2} / 2\right)$. In contrast, as the SL direction rotates anticlockwise to the armchair, there is a change of multiple $a_{2}$ for the vertical component of $\mathbf{T}$ but the horizontal component maintains unchanged due to the data for the boundaries being distributed in the right periphery of the second " $U$." The resulting series of boundary chiral numbers are shown in the third and fourth columns in Table I, respectively. Examples of crystal directions with chiral indexes $(4,2),(2,4)$, and $(1,5)$ from $\theta_{1}$ to $90^{\circ}$ are indicated in Fig. 1 by the thin blue and red solid lines, respectively. However, as shown by the colored stars in Fig. 2, the data for the three special boundary 
TABLE I. The 2D crystallographic directions of phosphorene with chiral indexes and angles on the two $U$ lines in Fig. 2.

\begin{tabular}{lccc}
\hline \hline $0^{\circ}-\theta_{1}$ & $\theta_{1}-\theta_{2}$ & $\theta_{2}-\theta_{3}$ & $\theta_{3}-90^{\circ}$ \\
Zigzag-SA & SA-SZ & SZ-SL & SL-armchair \\
$(m, n) \theta$ & $(m, n) \theta$ & $(m, n) \theta$ & $(m, n) \theta$ \\
\hline$(2,0) 0^{\circ}$ & $(3,1) 23.74^{\circ}$ & $(1,1) 52.84^{\circ}$ & $(1,3) 75.82^{\circ}$ \\
$\left(2 m^{\prime}+1,1\right) \rightarrow 0^{\circ}$ & $(4,2) 33.41^{\circ}$ & $\left(m^{\prime}, n^{\prime}+2\right) \rightarrow 52.84^{\circ}$ & $(1,5) 81.38^{\circ}$ \\
$\vdots$ & $\vdots$ & $\vdots$ & $\vdots$ \\
$(5,1) 14.78^{\circ}$ & $\left(m^{\prime}+2, n^{\prime}\right) \rightarrow 52.84^{\circ}$ & $(2,4) 69.24^{\circ}$ & $\left(1,2 n^{\prime}+1\right) \rightarrow 90^{\circ}$ \\
$(3,1) 23.74^{\circ}$ & $(1,1) 52.84^{\circ}$ & $(1,3) 75.82^{\circ}$ & $(0,2) 90^{\circ}$ \\
\hline \hline
\end{tabular}

directions, the zigzag, the SZ, and the armchair are outside the $U$-shape valleys. In fact, the nanostructures with these edges are the most stable and are favorably formed in experiments [45].

Moreover, when we cut a 2D phosphorene sheet along a certain crystal direction, it is found that two different manners exist for tangents to atoms, due to the two nonequivalent phosphorus atoms per primitive cell. Therefore, the direction $(1,1) 52.84^{\circ}$ is very particular, behind (beyond) which the boundary edge is nonbearded (bearded) when tangent to the lower atom, and vice versa when tangent to the upper atom of the primitive cell. That is to say, the SZ is the demarcation line between the with and without beard conditions for the boundary edges. Further, for the cutting manner shown by the solid lines in Fig. 1, all the boundary edges have an obvious zigzaglike edge atomic morphology except along the $\mathrm{SZ}$ and armchair directions. Whether a boundary edge is bearded or nonbearded depends on the number of dangled bonds of the outermost atoms. For bearded (nonbearded) boundary edges, the number of dangled bonds of the outermost atoms is two (one) per atom.

Actually, when we consider the phosphorene nanostructures, the effect of the $z$ direction on the characterization must be taken into account. This effect can be reflected by the fact that there are two kinds of arrangement for the outermost atoms along each crystallographic direction. According to our definition for $\mathbf{T}$, the chiral indexes satisfying $m+n=$ even have defined all the directions for a phosphorene sheet shown in Fig. 1 . When both $m$ and $n$ are odd, however, the outermost phosphorus atoms along the direction are alternately located at the upper and lower sublayers. So the supercell width of the nanoribbon cutting along this direction is twice $T$. A typical example of this case is shown by the thick-red-solid-line segment along the $\mathrm{SA}(3,1)$ direction in Fig. 1. In comparison, when both $m$ and $n$ are even, the outermost atoms are located at the same sublayer. In this case, the supercell width is just $T$ [see the $(4,2)$ direction]. The detailed classification of the nanoribbons is discussed in the Supplemental Material [55].

\section{B. PNRs and the PNR-based Z-shaped junction}

According to the structural characterization of phosphorene in the previous subsection, one can principally obtain PNRs with different edge morphologies by cutting a phosphorene sheet along different crystallographic directions. In addition to the extensively studied ZPNR and APNR [13,29], the ribbons made by cutting along the other three regional boundary directions $\mathrm{SA}(3,1), \mathrm{SZ}(1,1)$, and $\operatorname{SL}(1,3)$, indicated by the thick solid lines in Fig. 1, are denoted as $(3,1) \mathrm{nbPNR},(1,1) \mathrm{bPNR}$, and $(1,3) \mathrm{bPNR}$, respectively, where "bPNR" or "nbPNR" denotes a ribbon with or without bearded edges. On the other hand, the ribbons made by cutting along $\mathrm{SA}(3,1), \mathrm{SZ}(1,1)$, and SL $(1,3)$ according to the dashed lines in Fig. 1 are denoted as $(3,1) \mathrm{bPNR},(1,1) \mathrm{nbPNR}$, and $(1,3) \mathrm{nbPNR}$, respectively. These six types of PNRs are sketched in Figs. 3(a)-3(f), respectively, where the red dashed parallelogram in each PNR indicates its minimum periodical supercell. Since their outermost atoms along these ribbon edges are alternately located at the upper and lower sublayers, their supercell width is twice $T$ for both bearded and nonbearded edges. Among these ribbons, only $(3,1) \mathrm{PNR}$ and $(1,1) \mathrm{PNR}$ have been previously studied [14]. For the $(1,3)$ PNRs along SL, the chiral angle is $75.82^{\circ}$ and the edge morphology is more likely to be an APNR, which is obviously different from the $(3,1)$ PNRs along SA $\left(23.74^{\circ}\right)$ near the zigzag edge. In addition, these ribbons obviously do not have mirror symmetry, as do the normal ZPNR and APNR [13]. As mentioned in the above subsection, the ribbons cutting along the SA and SL solid lines have a periodic zigzaglike edge morphology. Importantly, this edge morphology is destroyed for the ribbons along the dashedline directions. Therefore, in order to reflect the particular edge atomic morphology, as shown in Figs. 3(a)-3(f), we have to choose different red-dashed-line parallelograms as the supercells for $(3,1) \mathrm{nbPNR},(1,1) \mathrm{bPNR},(1,3) \mathrm{bPNR}$, $(3,1) \mathrm{bPNR},(1,1) \mathrm{nbPNR}$, and $(1,3) \mathrm{nbPNR}$, respectively.

The stability of PNRs is an important issue, which has been previously studied by calculating the free energy $[36,43]$. The stability for $(3,1)$ PNR and $(1,1)$ PNR has been studied in previous work, which implies that the nonbearded ribbon is more stable. Therefore, in this work, 
(a) $(3,1) \mathrm{nbPNR}$

(b) $(1,1) \mathrm{bPNR}$

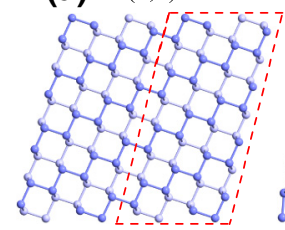

(d) $(3,1) \mathrm{bPNR}$
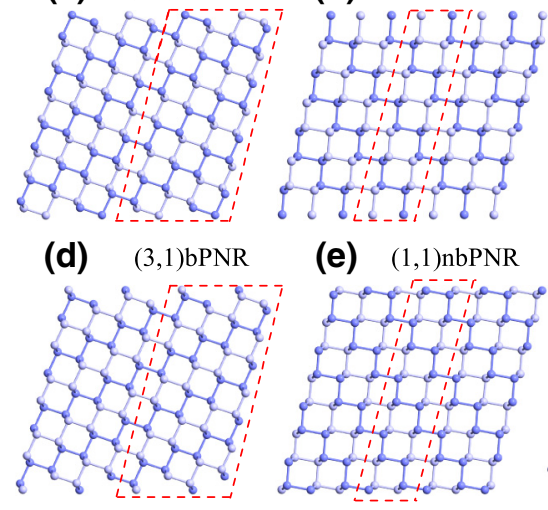

(e) $(1,1) \mathrm{nbPNR}$

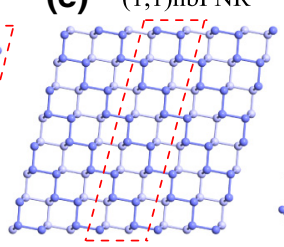

(c) $(1,3)$ bPNR

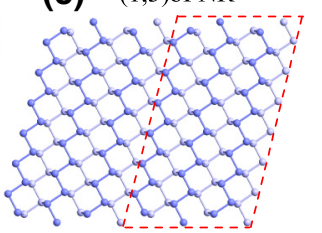

(f) $(1,3)$ nbPNR

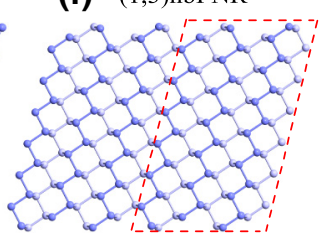

FIG. 3. Illustrations of (a) $(3,1) n b P N R$, (b) $(1,1) \mathrm{bPNR}$, (c) $(1,3)$ bPNR, (d) $(3,1) b P N R$, (e) $(1,1) n b P N R$, and (f) $(1,3) n b P N R$, where the red-dashed-line parallelogram in each PNR indicates its minimum periodical supercell.

we just compare the stability of the three ribbons in the nonbearded case and we also study the difference in stability between $(1,3)$ bPNR and $(1,3) n b P N R$. Using a firstprinciples calculation based on density-functional theory (DFT) [33], we verify the binding energies of the four narrow ribbons shown in Figs. 3(a), 3(c), 3(e), and 3(f). The order of the stability for these ribbons is $(1,1) n b P N R$, $(3,1) n b P N R,(1,1) n b P N R,(1,3) n b P N R$, and $(1,3) b P N R$, with the width being approximately $2 \mathrm{~nm}$. With an increase of the width, the ribbons will become more stable, with lower binding energies.

Next, we propose a two-terminal Z-shaped junction based on PNRs as shown in Fig. 4, where the electrodes of the junction are two semi-infinite ZPNRs with the same width of approximately $100 \mathrm{~nm}$ and the central scattering region is a Z-shaped PQD that contains a segment of PNR with different tilting angles. The tilting angle $\theta$ of the PQD corresponding to the chiral angle varies from 0 to $90^{\circ}$; as a result, the two hypotenuse edges of the PQD sweep over all possible geometrical morphologies. The enlarged typical edge geometries of the ribbons in Figs. 3(a)-3(c) [Figs. 3(d)-3(f)] are shown on the lefthand (right-hand) side of Fig. 4. This type of homogeneous junction may be etched on a sample sheet of 2D materials as a development of experimental technology and it has been extensively studied for graphene, silicene, $h$-BN, and so on $[56,57]$. However, for phosphorene, the edge (state) effect has only been considered for quantum-dot and ring structures [58,59], although it is primarily the PNR-based Z-shaped junction that has been studied [60]. In this work, we further study this Z-shaped PNR junction to explore the influence of the variation in edge geometrical morphology (corresponding to different tilting angles) on the transport properties. The significance is that the connection between the different parts of the homogeneous junction does not destroy the original lattice structure.
This may be impossible for inhomogeneous heterojunctions with electrodes made of another material, because there is always a lattice mismatch. Furthermore, the Zshaped junction allows for a short ribbon with all possible edges embedded in the junction, in which the existence of the resonant-tunneling phenomenon [61] can be expected.

\section{Computational details}

The numerical calculations here are performed by using the KWANT code based on the TB Hamiltonian and the atomistic quantum-transport simulations are based on the scattering-matrix approach from solving the wave function [50-52]. Compared with the conventional first-principles calculations [33], this method can calculate large nanostructures matching the usual experimentally reachable sample size up to sub-100-nm scales with better precision [48]. This advantage enables us to truly explore the electron structure and transport properties for the phosphorene nanostructures with different edge morphologies realized in our experiments and to provide a fundamental understanding in preparation for the design of nanodevices $[39,62]$.

In specification, the ribbons shown in Fig. 3 and the junction shown in Fig. 4, whether the system with and without bearded edges is considered, respectively. And the stability are analyzed using the first-principles approach. In the calculation, the scale of the systems is adapted by about $10-30 \mathrm{~nm}$ to meet the current experimental development [62], which is large enough to show a good illustration of the credibility and feasibility for the calculated results. This scale is more suitable for using KWANT, a PYTHON package for numerical quantum-transport calculations. We use a TB model based on the quasiparticle orbit to describe the low-energy electrons in phosphorene [54]. The parameters of the TB model are obtained by fitting its low-energy-band structure of phosphorene to the one computed using the density-functional theory- (DFT) $G W$ approach [54] and it has been shown that the fitted TB

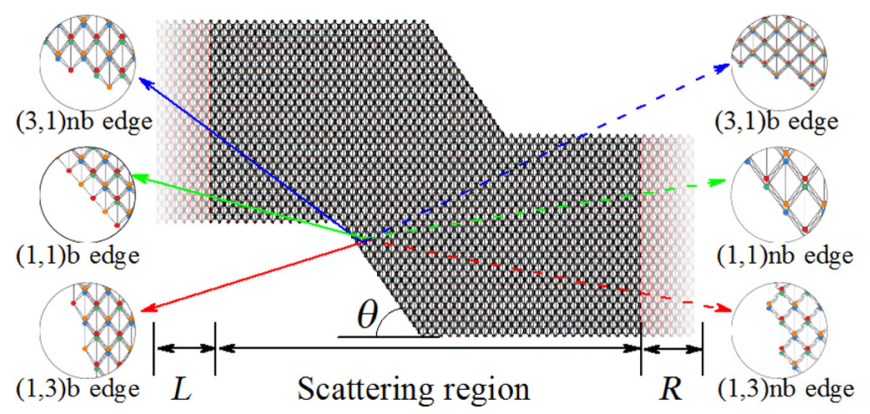

FIG. 4. A schematic illustration of the PNR-based Z-shaped junction, where the left-hand and right-hand enlarged views show the six types of edge geometric morphologies in the central scattering region with variation of the tilting angle [corresponding to Figs. 3(a)-3(f)]. 
model can reproduce the band structure of phosphorene in the low-energy regime well compared to the DFT- $G W$ approach [54].

The formal TB Hamiltonian for the considered system is

$$
H=\sum_{i} \varepsilon_{i} c_{i}^{\dagger} c_{i}+\sum_{i \neq j} t_{i j} c_{i}^{\dagger} c_{j}
$$

where the summation runs over all lattice sites, $\varepsilon_{i}$ is the on-site energy at site $i, t_{i j}$ is the hopping energy between sites $i$ and $j$, and $c_{i}^{\dagger}\left(c_{j}\right)$ is the creation (annihilation) operator of an electron at site $i(j)$. It has been shown that five hopping parameters (see Fig. 1) are enough to describe the electronic band structure of phosphorene with hopping energies $t_{1}=-1.220 \mathrm{eV}, t_{2}=3.665 \mathrm{eV}, t_{3}=-0.205$ $\mathrm{eV}, t_{4}=-0.105 \mathrm{eV}$, and $t_{5}=-0.055 \mathrm{eV}$ [54]. Starting from the Hamiltonian, we can calculate the local density of states (LDOS) using the following formula:

$$
\operatorname{LDOS}(E)=\frac{1}{c \sqrt{2 \pi}} \sum_{n}\left|\Psi_{n}(r)\right|^{2} e^{-\left(E_{n}-E\right)^{2} / 2 c^{2}},
$$

where $c$ is a broadening parameter and $\Psi_{n}(r)$ and $E_{n}$ are the eigenfunction and eigenvalue, respectively, in which $n$ denotes the energy-band index and $r$ the atom position. Further, the conductance of the junction at zero temperature can be calculated by using the Landauer formula [63]

$$
G=\frac{2 e^{2}}{h} \int d E\left(-\frac{\partial f_{0}}{\partial E}\right) T(E),
$$

where $f_{0}(E)=1 /\left[e^{\left(E-E_{F}\right) / k_{B} T}+1\right]$ is the equilibrium distribution function with Fermi energy $E_{F}$ and $T(E)=$ $\operatorname{Tr}\left[\Gamma_{L}(E, V) G(E, V) \Gamma_{R}(E, V) G^{\dagger}(E, V)\right]$ - in which $L$ and $R$ label the left and right electrodes of the system, respectively - is the transmission coefficient through the junction from electrode $L$ to electrode $R$, with $G\left(G^{\dagger}\right)$ being the retarded (advanced) Green function of the central region and $\Gamma_{L / R}$ being the coupling matrix between the scattering region and the left or right electrode.

\section{NUMERICAL RESULTS AND DISCUSSIONS}

\section{A. Electronic structure for PNRs}

By solving the difference Schrödinger equation corresponding to the Hamiltonian on the proper basis for the supercell adopted in Fig. 3 and applying the Bloch theorem, the $k$-dependent Hamiltonian for a PNR can be written as $H(k)=H_{0,0}+H_{0,1} e^{i k a}+H_{0,1}^{\dagger} e^{-i k a}$ in the form of an $(N \times N)$ dimensional matrix. Here, $N$ is the number of atoms in the supercell drawn by the red dashed line in Fig. 3, $H_{0,0}$ is the matrix of the central cell, $H_{0,1}$ is the coupling matrix with the right-hand adjacent cell, and $a$ is the length between two nearest-neighbor cells. Diagonalizing this $k$-dependent Hamiltonian, we can obtain the band spectrum and the corresponding eigenwave functions. Following the procedure described in the last subsection, we can also obtain the conductance and the LDOS for the system.

The calculated energy band (left-hand panels) and conductance (right-hand panels) are shown in Figs. 5(a)-5(d) for the four most stable $(3,1) n b P N R,(1,1) n b P N R$, $(1,3) \mathrm{bPNR}$, and $(1,3) \mathrm{nbPNR}$ with widths around $4 \mathrm{~nm}$, respectively. Also, the choice of the supercells has consequently taken the effect of the $z$ direction into consideration. In each figure, the embedded inset in the right-hand panel is the LDOS at the energy indicated by the red dot in the corresponding left-hand panel. First, for the band structure of $(3,1)$ nbPNR shown in Fig. 5(a), the twofolddegenerate edge bands indicated by the red dashed and blue solid lines pass through the Fermi level exhibiting the metallic property, while $(1,1) n b P N R$ is a direct-bandgap semiconductor as shown in Fig. 5(b). This recovers the results of the previous straightforward calculation for these two types of ribbon [14]. However, for the discovered $(1,3) \mathrm{bPNR}$, the twofold-degenerate edge bands around the Fermi level are also detached from the bulk bands. This is similar to the band structure of $(3,1) n b P N R$, but the bulk band (thin black solid line) and its position relating to the edge state are still significantly different. To further confirm the existence of the edge states, we calculate the LDOS at the energy marked by the red dots on the edge bands; the thumbnail of LDOS embedding in the right-hand panels depicts the real-space localization of electrons. From the LDOS for $(3,1) \mathrm{nbPNR}$ and $(1,3) \mathrm{bPNR}$, we observe the obvious electron clouds to be mainly localized at the unsaturated phosphorus atoms in the zigzaglike edges of the ribbons, which results from the lone pair electrons at the edges [16]. In contrast, for $(1,1) n b P N R$, the electron states at the conduct band minimum (CBM) are not contributed by the atoms at the edges of the ribbons.

Further, the distinctive electronic properties of PNRs have a direct consequence on the transport properties. As shown in each right-hand panel of Figs. 5(a)-5(d), the conductance displays a clear stepwise structure. The quantized conductance plateaus follow the sequence $G=n e^{2} / h$, with $n$ denoting positive integers. For $(3,1) n b P N R$ and $(1,3) \mathrm{bPNR}$, when the energy is within the band gap around the Fermi level, their conductance has a quantized plateau, which is clearly induced by the edge states. However, neither $(1,1)$ nbPNR nor $(1,3)$ nbPNR have a quantized plateau near the Fermi level, because there are no edge states near the Fermi level. On the other hand, we need to understand the effect of a PNR with a bearded or nonbearded edge on the electronic structure. Previous studies have shown that for the beard-ZPNR [or $(2,0) b P N R],(3,1) b P N R$, and $(1,1) \mathrm{bPNR}$, the band structure is essentially different 


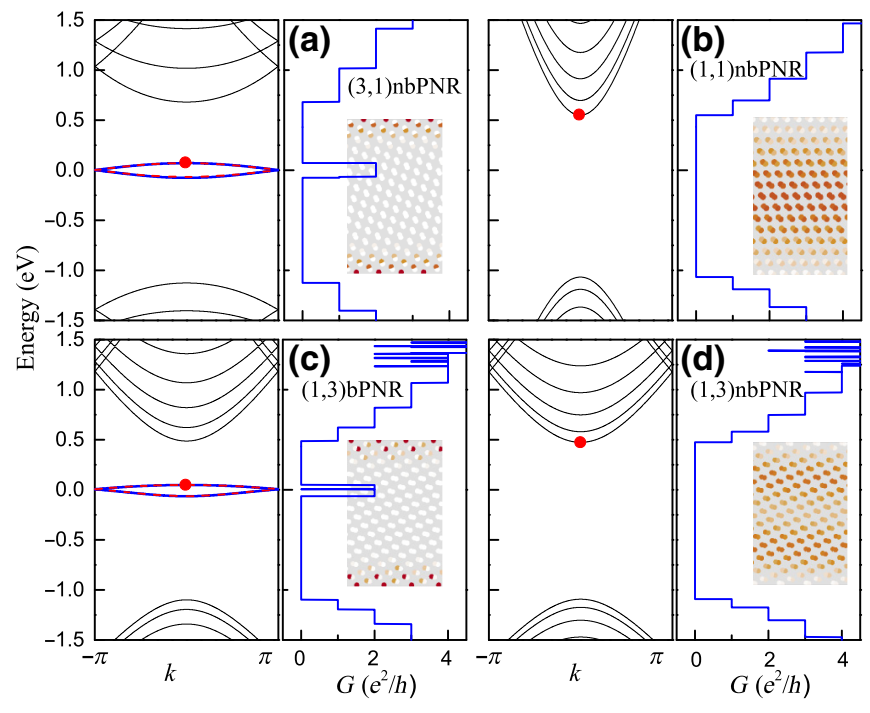

FIG. 5. The calculated energy band (left-hand panels) and conductance (right-hand panels) for (a) $(3,1) \mathrm{nbPNR}$, (b) $(1,1) n b P N R$, (c) $(1,3)$ bPNR, and (d) $(1,3)$ nbPNR with widths around $4 \mathrm{~nm}$, respectively, where the embedded inset in each right-hand panel is the LDOS at the energy indicated by the red dot in the corresponding left-hand panel.

from that for the nonbearded counterpart, especially with regard to whether or not edge states exist [14,29]. For $(2,0)$ bPNR and $(3,1)$ bPNR, we see that the bulk bands remain unchanged, but the edge states disappear. For the discovered $(1,3) \mathrm{bPNR}$, an edge band occurs around the Fermi level. However, $(1,3) b P N R$ is special due to the original bearded edges and its band structure is characterized by the significant edge states, as shown in Figs. $3(\mathrm{c})$ and 5(c). Interestingly, when we translate the edge outward by one atom for $(1,3)$ bPNR, we then obtain $(1,3) n b P N R$, as shown in Fig. 3(f). As a result, as shown in Fig. 5(d) in comparison with Fig. 5(c), although its bulk bands are unchanged, the edge states disappear. Hence it becomes a direct-band-gap semiconductor like the APNR and $(1,1) n b P N R$.

For a pristine PNR, in summary, the presence or absence of a bearded edge determines the existence of the zigzaglike edge morphology resulting in the edge-state bands. The electronic localization caused by the edge zigzaglike atomic configuration may be due to the that phosphorene is a second-order topological insulator [64], which is still an open question. The PNRs with possible edges along the directions given by the solid lines in Fig. 1 all have edge states near the Fermi energy, except for the armchair edge. Along these directions, there is always a periodic zigzaglike edge geometry, except for $(1,1) \mathrm{bPNR}$, the edge states of which are caused by the unsaturated atoms. Therefore, the $(1,1) \mathrm{SZ}$ crystallographic direction is a demarcation line, behind (beyond) which are the PNRs without (with) bearded edges, or vice versa for an alternative tangent manner along the same direction. Within the chiral angle range of $0-\theta_{2}$ (below the SZ), all the PNRs without a bearded edge have a zigzaglike edge morphology, but this edge morphology is absent for the corresponding bearded PNRs. In this case, the electrons are not localized on the outermost atoms, which results in no edge states. For the $\theta_{2}-90^{\circ}$ range, in contrast, the zigzaglike edge morphology of the bearded PNRs derives originally from the dangling phosphorus atoms, but for nonbearded PNRs this property disappears due to the saturation of the dangling atoms. This conclusion is consistent with that for graphene [41].

\section{B. Transport properties of the Z-shaped junction}

The proposed Z-shaped junction in Fig. 4 consists of two semi-finite ZPNR electrodes and the central scattering region of a Z-shaped PQD. As the degree of torsion of the junction is varied, the tilting angle of the center PQD can be altered from 0 to $90^{\circ}$; hence the whole junction is changed from a standard ZPNR to an APNR. However, in this process the two edges of the PQD sweep over all possible geometrical morphologies described in Secs. II B (see also Fig. 1 and Table I). As a result, the electronic band structure of the PQD varies alternatively between the presence and absence of edge states near the Fermi energy and the whole junction is consequently either conducting or semiconducting. In order to explore the variation of transport for the junction, in Figs. 6(a)-6(f), we first show the calculated conductances (in units of $e^{2} / h$ ) for the junction with PQD edges along the three regional boundaries shown in the two sides of Fig. 4, with $(3,1) \mathrm{nb},(1,1) \mathrm{b},(1,3) \mathrm{b}$, $(3,1) \mathrm{b},(1,1) \mathrm{nb}$, and $(1,3) \mathrm{nb}$ edges, respectively. Apparently, as shown in Figs. 6(a)-6(c), the junction of a PQD with the $(3,1) n b,(1,1) b$, and $(1,3) b$ edges, respectively,

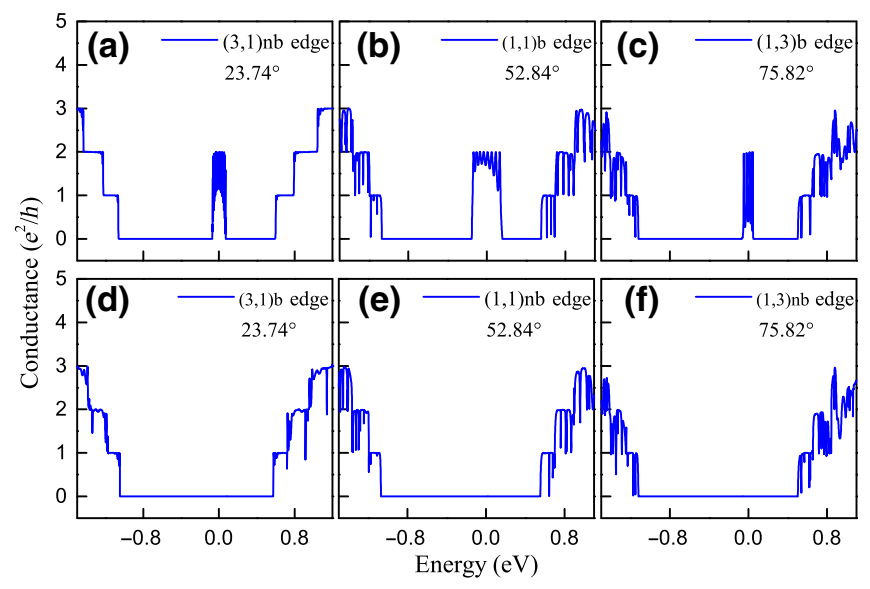

FIG. 6. The conductance (in units of $e^{2} / h$ ) spectrum of the Z-shaped junction, where the central scatter PQD with (a) the $(3,1) \mathrm{nb}$ edge, (b) the $(1,1) \mathrm{b}$ edge, (c) the $(1,3) \mathrm{b}$ edge, (d) the $(3,1) \mathrm{b}$ edge, (e) the $(1,1) \mathrm{nb}$ edge, and (f) the $(1,3) \mathrm{nb}$ edge correspond to Figs. 3(a)-3(f), respectively. 


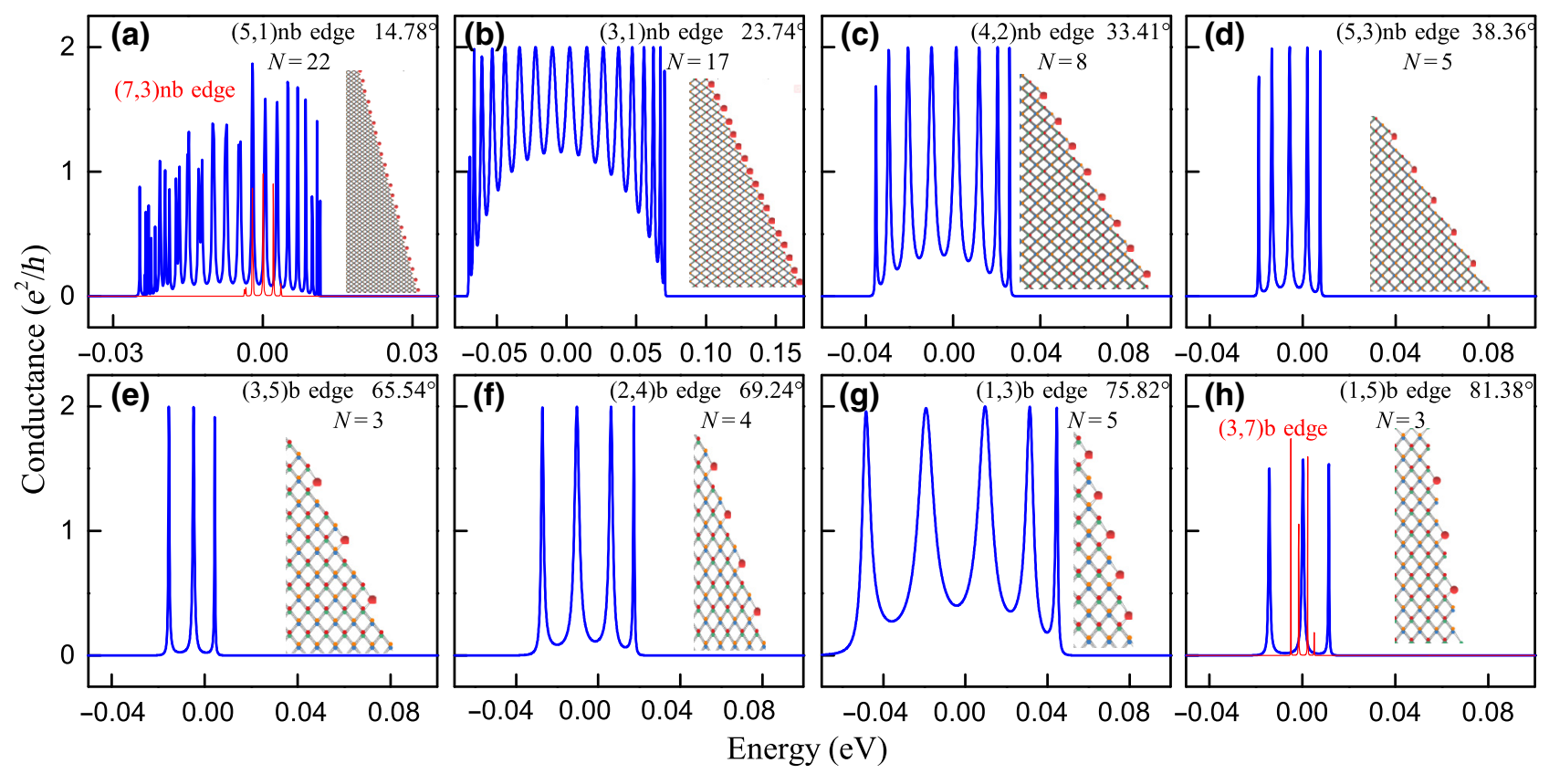

FIG. 7. The oscillation peaks on the conductance terrace (in units of $e^{2} / h$ ) within the low-energy range for the Z-shaped junction with an increase of the titling (chiral) angle $\theta$, for (a) the $(5,1) n b$ edge, (b) the $(3,1) \mathrm{nb}$ edge, (c) the $(4,2)$ nb edge, (d) the $(5,3)$ nb edge, (e) the $(3,5) b$ edge, (f) the $(2,4) b$ edge, $(\mathrm{g})$ the $(1,3) \mathrm{b}$ edge, and $(\mathrm{h})$ the $(1,5) \mathrm{b}$ edge. The thin red lines in $(\mathrm{a})$ and $(\mathrm{h})$ are the conductances for the $(7,3) \mathrm{nb}$ and $(3,7) \mathrm{b}$ edges, respectively. The insets show the corresponding PQD edge geometries, where the red dots denote the outermost phosphorus atoms along the zigzaglike edge and integer $N$ is the number of outermost atoms.

exhibits a conductance plateau of $2 e^{2} / h$ with oscillations within the energy ranges -0.072 to $+0.072 \mathrm{eV},-0.153$ to $+0.156 \mathrm{eV}$, and -0.095 to $+0.053 \mathrm{eV}$, respectively, due to the edge states [14]. On the contrary, the junction of a PQD with $(3,1) b$, $(1,1) n b$, and $(1,3) n b$ edges, as shown in Figs. 6(d)-6(f), displays almost zero conductance because of the obvious band gap [14]. The result indicates that the edge states of the PQD also play an important role in transport for a Z-shaped junction. This interesting transport property has also been reported for a planar homogenous junction formed by graphite ribbons with different edges [61].

Next, to find the origin of the conductance oscillating peaks, in Fig. 7 we present the low-energy conductance spectra for the junction with $\theta$ in ascending order from 14.78 to $81.38^{\circ}$, within which the PQDs along the directions of the $(5,1) \mathrm{nb},(3,1) \mathrm{nb},(4,2) \mathrm{nb},(5,3) \mathrm{nb},(3,5) \mathrm{b}$, $(2,4) \mathrm{b},(1,3) \mathrm{b}$, and $(1,5) \mathrm{b}$ edges are selected, respectively. These eight edges all have an obvious zigzaglike geometry, with enlarged views of the edges inserted in each figure. Consistent with the previous the band structure for PNRs with different edges, in Figs. 7(a)-7(d) the conductance spectra for the PQD with four different nonbearded edges all have an oscillating terrace provided by the edge states around the Fermi level. Since the function of the outermost atoms along the zigzaglike configuration is similar to those of the actual zigzag edge, they are used as a medium atom for electron transport [29]. On the other hand, the spectra shown in Figs. 7(e)-7(h), respectively, for bearded
$(3,5),(2,4),(1,3)$, and $(1,5)$ edges have a similar feature, with an oscillating terrace provided by the edge states. Although these edges with a tilting angle close to $90^{\circ}$ result in an edge atomic configuration similar to the armchair type, the bearded SL and its derivative edges still form a periodic zigzaglike configuration due to the outermost dangling phosphorus atoms. This function of the bearded edge for PNRs has also been demonstrated previously $[14,29]$.

When the hypotenuse of a PQD has an edge behind or beyond the SZ direction, such as in the case of the $(3,1) \mathrm{nb}$ and $(1,3) \mathrm{b}$ edges, the junctions exhibit similar transport behavior due to the existence of zigzaglike edge geometry. This is because the outermost phosphorus atom along the zigzaglike edges of the PQD forms an electronic bound-state [64], which is expressed in the energy band as discrete energy levels. These discrete energy levels cause the orphaned electrons at the edge to be hopped, to produce an oscillating form of transport behavior. The function of the outermost atoms along the edges can also be reflected well in the conductance spectrum. Interestingly, as clearly shown in Figs. 7(a)-7(h), the number $(N)$ of the terrace oscillating peaks exactly matches the number of outermost phosphorus atoms indicated by the red dots within a hypotenuse edge of a PQD, which is also related to the number of minimum-period length $T$ along a hypotenuse edge of a PQD because the electrons are localized at each zigzag configuration regardless of the sublayer. Therefore, for a Z-shaped junction with a certain 


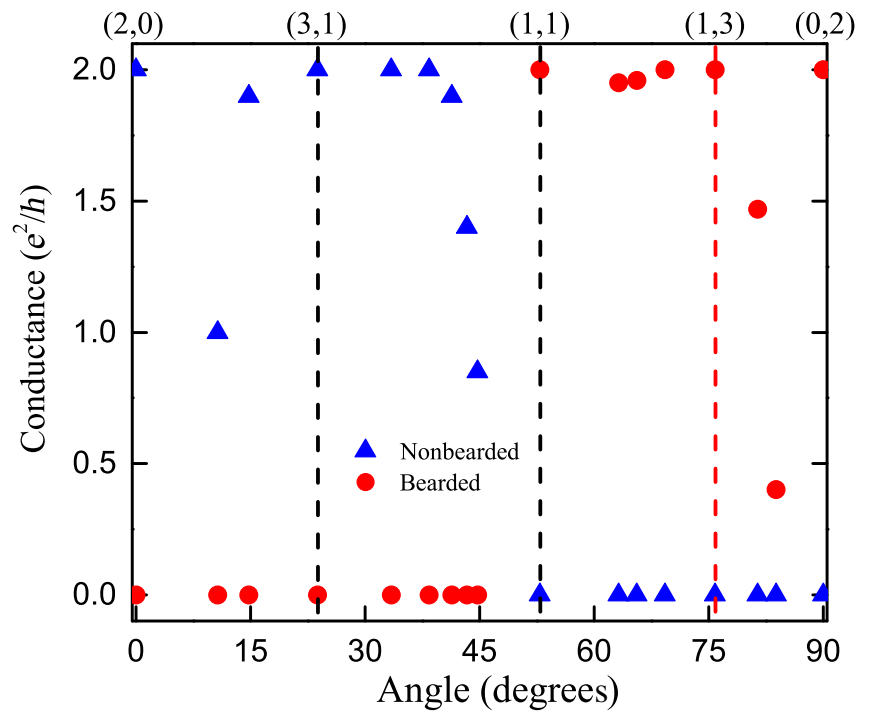

FIG. 8. The conductance of the junction as a function of the tilting angle with $|\mathbf{T}|$ less than $20 \AA$, where the hypotenuse of the central part with and without a bearded edge is indicated by the red spheres and the blue triangles, respectively, and the vertical dashed lines imply the three particular regional boundaries.

size, as the edge of the central PQD continuously sweeps over all possible values of $\theta$, the value of $N$ is varied. In addition to the above eight tilting angles, there are many other angles, as indicated by the different-colored and shaped dots in Fig. 2. The conductance spectrum of a junction with these edge boundaries also exhibits oscillating characteristics in the low-energy regime, as shown by the thin red lines in Figs. 7(a) and 7(h). For large $T$, the zigzaglike edge may have more inner structures in comparison with those for edges with small $T$. Further, these atomic configurations also produce various scatters that can cause severe interference to the oscillating transport of the electrons, making the discrete levels degenerate and reducing the corresponding conductance. For example, in Fig. 7(a), due to the degeneracy of the energy level and the scattering of the bearded edge atomic configurations, the number of conductance peaks is less than that of the outermost phosphorus atoms and the conductance value is also less than $2 e^{2} / h$. On the other hand, the value of $T$ also limits the conductance. By more calculations, we find that the conductance of the junctions with $T$ less than $20 \AA$ is effective in the low-energy regime; otherwise, the conductance value is extremely small as $T$ is beyond this critical value.

Finally, the data for the calculated conductance versus the titling angle for our Z-shaped junction, the central PQD of which has more possible edges with $T$ less than the critical value of $20 \AA$, are summarized in Fig. 8, where the blue (red) triangles (spheres) for the nonbearded (bearded) case and the vertical dashed lines indicate the three particular regional boundaries. As the titling (chiral) angle $\theta$ varies from 0 to $90^{\circ}$, we can see that the $\mathrm{SZ}(1,1)$ at $52.84^{\circ}$ is more special, behind (beyond) which the conductance is nonzero (zero) for the junction with nonbearded edges. The situation for the junction with bearded edges is just the opposite of the nonbearded case and the two cases are complementary. In addition, more information can be obtained from the phaselike diagram shown in Fig. 8. For instance, the conductance of the junction with $\theta$ ranging from 0 to $90^{\circ}$ depends sensitively on the module length $T$ between 0 and $2 e^{2} / h$. Near the three regional boundaries $0^{\circ}$ (zigzag), $52.84^{\circ}$ (SZ), and $90^{\circ}$ (armchair), $T$ is larger, which results in a decreasing conductance. This result is consistent with the distribution of $T$ near the bottom of the "U" in Fig. 2. If $T$ is beyond this critical value, the conductance tends to zero, although there are still edge channels for the junction in the low-energy regime.

\section{SUMMARY AND CONCLUSION}

We theoretically study the 2D crystallographic characterization of an anisotropic phosphorene and its applications in nanostructures. A chiral number (vector) or chiral angle is defined to uniformly describe the planar crystallographic directions on a phosphorene. As the chiral angle increases from $0^{\circ}$ to $90^{\circ}$, the edge geometry along the boundary varies from zigzag to armchair and sweeps over all possible edge morphologies during the process. We find that a phosphorene can be divided into four fanshaped regions determined by boundary chiral angles $0^{\circ}$, $\theta_{1}\left(23.74^{\circ}\right), \theta_{2}\left(52.84^{\circ}\right), \theta_{3}\left(75.82^{\circ}\right)$, and $90^{\circ}$, among which the characteristics are different each other. Therefore, by cutting a phosphorene along these five regional boundary directions, one can obtain the previously studied ZPNR, SAPNR, SZPNR, and APNR. This leads to the definition of a PNR that has an edge atomic configuration with SL edge geometry along $\theta_{3}$. Essentially, the SL direction is similar to the SA one, with a periodic zigzaglike edge morphology supporting edge states. Further, we then demonstrate the variation in electronic property for PNRs with different edge atomic geometries by using the KWANT TB code, in which particular attention is paid to the SLPNR, which intrinsically has twofold-degenerate edge states. In addition, in each regional boundary direction, the origin of the bearded and nonbearded edges is discussed and related to the existence of the edge states. We find that the $(1,1)$ direction is the dividing line, below (beyond) which the ribbon with has nonbearded (bearded) edges.

Second, we propose a two-terminal Z-shaped homogenous junction based on a PNR and calculate its transport properties by using the scattering-matrix approach to treat large enough systems that are comparable with experimental samples. The two electrodes of the junction are semi-infinite ZPNRs with the same width of approximately $100 \mathrm{~nm}$, where the central scattering region of a segment of PNR with edge-tilting angles from 0 to $90^{\circ}$ is sandwiched in between. As a consequence, the two hypotenuse 
edges of the central PQD sweep over all possible geometrical morphologies. Interestingly, the conductance spectrum depends sensitively on the tilting angle of the Z-shaped junction. For the junctions of PQDs with edge states, the transport exhibits a conductance terrace with resonant oscillation in the low-energy regime; otherwise, electron transport is blocked due to the absence of edge states. Remarkably, the number of conductance oscillating peaks is exactly equal to the number of outermost phosphorus atoms in the zigzaglike edges of the center PQD, since the discrete energy levels of the zigzaglike atomic configurations provide the transport channels for the electrons. The findings here may be extended to the group-VA 2D materials, such as 2D group-VA arsenene, antimonene, and bismuthene, to observe the electronic properties of nanostructures in experiments and provide a reference for the preparation of better nanodevices.

\section{ACKNOWLEDGMENTS}

This work was supported by the National Natural Science Foundation of China (Grants No. 11774085, No. 11804092, and No. 11704118), the China Postdoctoral Science Foundation funded project (Grants No. BX20180097 and No. 2019M652777), and the Hunan Provincial Natural Science Foundation of China (Grant No. 2019JJ40187).

[1] A. K. Geim and K. S. Novoselov, The rise of graphene, Nat. Mater. 6, 183 (2007).

[2] Likai Li, Yijun Yu, Guo Jun Ye, Qingqin Ge, Xuedong Ou, Hua Wu, Donglai Feng, Xian Hui Chen, and Yuanbo Zhang, Black phosphorus field-effect transistors, Nat. Nanotech. 9, 372 (2014).

[3] Han Liu, Adam T. Neal, Zhen Zhu, Zhe Luo, Xianfan Xu, David Tománek, and Peide D. Ye, Phosphorene: An unexplored 2D semiconductor with a high hole mobility, ACS Nano 8, 4033 (2014).

[4] Fengnian Xia, Han Wang, and Yichen Jia, Rediscovering black phosphorus as an anisotropic layered material for optoelectronics and electronics, Nat. Commun. 5, 4458 (2014).

[5] Steven P. Koenig, Rostislav A. Doganov, Hennrik Schmidt, A. H. Castro Neto, and Barbaros Özyilmaz, Electric field effect in ultrathin black phosphorus, Appl. Phys. Lett. 104, 103106 (2014).

[6] Michele Buscema, Dirk J. Groenendijk, Sofya I. Blanter, Gary A. Steele, Herre S. J. van der Zant, and Andres Castellanos-Gomez, Fast and broadband photoresponse of few-layer black phosphorus field-effect transistors, Nano Lett. 14, 3347 (2014).

[7] Xi Ling, Han Wang, Shengxi Huang, Fengnian Xia, and Mildred S. Dresselhaus, The renaissance of black phosphorus, PNAS 112, 4523 (2015).

[8] Rishabh Jain, Rekha Narayan, Suchithra Padmajan Sasikala, Kyung Eun Lee, Hong Ju Jung, and Sang Ouk Kim, Phosphorene for energy and catalytic application-filling the gap between graphene and 2D metal chalcogenides, 2D Mater. 4, 042006 (2017).

[9] Pengfei Chen, Neng Li, Xingzhu Chen, Wee-Jun Ong, and Xiujian Zhao, The rising star of 2D black phosphorus beyond graphene: Synthesis, properties and electronic applications, 2D Mater. 5, 014002 (2018).

[10] Chenggang Tao, Liying Jiao, Oleg V. Yazyev, Yen-Chia Chen, Juanjuan Feng, Xiaowei Zhang, Rodrigo B. Capaz, James M. Tour, Alex Zettl, Steven G. Louie, Hongjie Dai, and Michael F. Crommie, Spatially resolving edge states of chiral graphene nanoribbons, Nat. Phys. 7, 610 (2011).

[11] Y. Li, Z. Zhou, S. Zhang, and Z. Chen, $\mathrm{MoS}_{2}$ nanoribbons: High stability and unusual electronic and magnetic properties, J. Am. Chem. Soc. 130, 16739 (2008).

[12] Mitchell C. Watts, Loren Picco, Freddie S. Russell-Pavier, Patrick L. Cullen, Thomas S. Miller, Szymon P. Bartuś, Oliver D. Payton, Neal T. Skipper, Vasiliki Tileli, and Christopher A. Howard, Production of phosphorene nanoribbons, Nature 568, 216 (2019).

[13] A. Carvalho, A. S. Rodin, and A. H. Castro Neto, Phosphorene nanoribbons, Europhys. Lett. 108, 47005 (2014).

[14] Marko M. Grujić, Motohiko Ezawa, Milan Ž. Tadić, and Francois M. Peeters, Tunable skewed edges in puckered structures, Phys. Rev. B 93, 245413 (2016).

[15] Young Woo Son, Marvin L. Cohen, and Steven G. Louie, Half-metallic graphene nanoribbons, Nature 444, 16 (2006).

[16] Vy Tran and Li Yang, Scaling laws for the band gap and optical response of phosphorene nanoribbons, Phys. Rev. B 89, 245407 (2014).

[17] Ajanta Maity, Akansha Singh, Prasenjit Sen, Aniruddha Kibey, Anjali Kshirsagar, and Dilip G. Kanhere, Structural, electronic, mechanical, and transport properties of phosphorene nanoribbons: Negative differential resistance behavior, Phys. Rev. B 94, 075422 (2016).

[18] Guang Yang, Shenglong Xu, Wei Zhang, Tianxing Ma, and Congjun $\mathrm{Wu}$, Room-temperature magnetism on the zigzag edges of phosphorene nanoribbons, Phys. Rev. B 94, 075106 (2016).

[19] C. Q. Han, M. Y. Yao, X. X. Bai, Lin Miao, Fengfeng Zhu, D. D. Guan, Shun Wang, C. L. Gao, Canhua Liu, Dong Qian, Y. Liu, and Jin-feng Jia, Electronic structure of black phosphorus studied by angle-resolved photoemission spectroscopy, Phys. Rev. B 90, 085101 (2014).

[20] Likai Li, Jonghwan Kim, Chenhao Jin, Guojun Ye, Diana Y. Qiu, Felipe H. da Jornada, Zhiwen Shi, Long Chen, Zuocheng Zhang, Fangyuan Yang, Kenji Watanabe, Takashi Taniguchi, Wencai Ren, Steven G. Louie, Xianhui Chen, Yuanbo Zhang, and Feng Wang, Direct observation of the layer-dependent electronic structure in phosphorene, Nat. Nanotech. 12, 21 (2017).

[21] Sherman Jun Rong Tan, Ibrahim Abdelwahab, Leiqiang Chu, Sock Mui Poh, Yanpeng Liu, Jiong Lu, Wei Chen, and Kian Ping Loh, Quasi-monolayer black phosphorus with high mobility and air stability, Adv. Mater. 30, 1704619 (2018).

[22] Xianghong Niu, Yunhai Li, Yehui Zhang, Qiang Li, Qionghua Zhou, Jin Zhao, and Jinlan Wang, Photooxidative degradation and protection mechanism of black 
phosphorus: Insights from ultrafast dynamics, J. Phys. Chem. Lett. 9, 5034 (2018).

[23] Xiaoying Zhou, Wen-Kai Lou, Dong Zhang, Fang Cheng, Guanghui Zhou, and Kai Chang, Effective $g$ factor in black phosphorus thin films, Phys. Rev. B 95, 045408 (2017).

[24] X. Y. Zhou, R. Zhang, J. P. Sun, Y. L. Zou, D. Zhang, W. K. Lou, F. Cheng, G. H. Zhou, F. Zhai, and Kai Chang, Landau levels and magnetotransport property of monolayer phosphorene, Sci. Rep. 5, 12295 (2015).

[25] Likai Li, Fangyuan Yang, Guo Jun Ye, Zuocheng Zhang, Zengwei Zhu, Wenkai Lou, Xiaoying Zhou, Liang Li, Kenji Watanabe, Takashi Taniguchi, Kai Chang, Yayu Wang, Xian Hui Chen, and Yuanbo Zhang, Quantum Hall effect in black phosphorus two-dimensional electron system, Nat. Nanotech. 11, 592 (2016).

[26] Ruge Quhe, Qiuhui Li, Qiaoxuan Zhang, Yangyang Wang, Han Zhang, Jingzhen Li, Xiuying Zhang, Dongxue Chen, Kaihui Liu, Yu Ye, Lun Dai, Feng Pan, Ming Lei, and Jing Lu, Simulations of Quantum Transport in Sub-5-nm Monolayer Phosphorene Transistors, Phys. Rev. Appl. 10, 024022 (2018).

[27] Gautam Gaddemane, William G. Vandenberghe, Maarten L. Van de Put, Shanmeng Chen, Sabyasachi Tiwari, Edward Chen, and Massimo V. Fischetti, Theoretical studies of electronic transport in monolayer and bilayer phosphorene: A critical overview, Phys. Rev. B 98, 115416 (2018).

[28] Paul Masih Das, Gopinath Danda, Andrew Cupo, William M. Parkin, Liangbo Liang, Neerav Kharche, Xi Ling, Shengxi Huang, Mildred S. Dresselhaus, Vincent Meunier, and Marija Drndic, Controlled sculpture of black phosphorus nanoribbons, ACS Nano 10, 5687 (2016).

[29] M. Ezawa, Topological origin of quasi-flat edge band in phosphorene, New J. Phys. 16, 115004 (2014).

[30] Esmaeil Taghizadeh Sisakht, Mohammad H. Zare, and Farhad Fazileh, Scaling laws of band gaps of phosphorene nanoribbons: A tight-binding calculation, Phys. Rev. B 91, 085409 (2015).

[31] D. J. P. de Sousa, L. V. de Castro, D. R. da Costa, and J. Milton Pereira, Jr. Boundary conditions for phosphorene nanoribbons in the continuum approach, Phys. Rev. B 94, 235415 (2016).

[32] S. K. Firoz Islam, Paramita Dutta, A. M. Jayannavar, and Arijit Saha, Probing decoupled edge states in a zigzag phosphorene nanoribbon via RKKY exchange interaction, Phys. Rev. B 97, 235424 (2018).

[33] Yi Ren, Fang Cheng, Z. H. Zhang, and Guanghui Zhou, Half metal phase in the zigzag phosphorene nanoribbon, Sci. Rep. 8, 2932 (2018).

[34] E. Taghizadeh Sisakht, F. Fazileh, M. H. Zare, M. Zarenia, and F. M. Peeters, Strain-induced topological phase transition in phosphorene and in phosphorene nanoribbons, Phys. Rev. B 94, 085417 (2016).

[35] Benliang Zhou, Benhu Zhou, Xiaoying Zhou, and Guanghui Zhou, Even-odd effect on the edge states for zigzag phosphorene nanoribbons under a perpendicular electric field, J. Phys. D: Appl. Phys. 50, 045106 (2017).

[36] Xiaoyu Han, Henry Morgan Stewart, Stephen A. Shevlin, C. Richard, A. Catlow, and Zheng Xiao Guo, Strain and orientation modulated bandgaps and effective masses of phosphorene nanoribbons, Nano Lett. 14, 4607 (2014).
[37] R. Ma, H. Geng, W. Y. Deng, M. N. Chen, L. Sheng, and D. Y. Xing, Effect of the edge states on the conductance and thermopower in zigzag phosphorene nanoribbons, Phys. Rev. B 94, 125410 (2016).

[38] Zahra Nourbakhsh and Reza Asgari, Charge transport in doped zigzag phosphorene nanoribbons, Phys. Rev. B 97, 235406 (2018).

[39] L. L. Li and F. M. Peeters, Quantum transport in defective phosphorene nanoribbons: Effects of atomic vacancies, Phys. Rev. B 97, 075414 (2018).

[40] S. Soleimanikahnoj and I. Knezevic, Pseudospin Electronics in Phosphorene Nanoribbons, Phys. Rev. Appl. 8, 064021 (2017).

[41] A. R. Akhmerov and C. W. J. Beenakker, Boundary conditions for Dirac fermions on a terminated honeycomb lattice, Phys. Rev. B 77, 085423 (2008).

[42] Te-Huan Liu, Yin-Chung Chen, Chun-Wei Pao, and ChienCheng Chang, Anisotropic thermal conductivity of $\mathrm{MoS}_{2}$ nanoribbons: Chirality and edge effects, Appl. Phys. Lett. 104, 201909 (2014).

[43] Ashwin Ramasubramaniam and Andre R. Muniz, Ab initio studies of thermodynamic and electronic properties of phosphorene nanoribbons, Phys. Rev. B 90, 085424 (2014).

[44] Yuanyue Liu, Fangbo Xu, Ziang Zhang, Evgeni S. Penev, and Boris I. Yakobson, Two-dimensional mono-elemental semiconductor with electronically inactive defects: The case of phosphorus, Nano Lett. 14, 6782 (2014).

[45] Liangbo Liang, Jun Wang, Wenzhi Lin, Bobby G. Sumpter, Vincent Meunier, and Minghu Pan, Electronic bandgap and edge reconstruction in phosphorene materials, Nano Lett. 14, 6400 (2014).

[46] Vladimir V. Arsoski, Marko M. Grujić, Nemanja A. Čkarić, Milanž. Tadić, and Francois M. Peeters, Normal and skewed phosphorene nanoribbons in combined magnetic and electric fields, Phys. Rev. B 96, 125434 (2017).

[47] Z. T. Lv, J. H. Gao, X. D. Zhang, and Z. T. Jiang, Coupling and manipulation of edge states in multilayer phosphorene nanoribbons, Physica E 94, 59 (2017).

[48] C. W. Groth, M. Wimmer, A. R. Akhmerov, and X. Waintal, KWANT: A software package for quantum transport, New J. Phys. 16, 063065 (2014).

[49] Shengli Zhang, Shiying Guo, Zhongfang Chen, Yeliang Wang, Hongjun Gao, Julio Gómez-Herrero, Pablo Ares, Félix Zamora, Zhen Zhu, and Haibo Zeng, Recent progress in 2D group-VA semiconductors: From theory to experiment, Chem. Soc. Rev. 47, 982 (2018).

[50] Yongjin Jiang and Liangbin Hu, Symmetry properties of spin currents and spin polarizations in multiterminal mesoscopic spin-orbit-coupled systems, Phys. Rev. B 75, 195343 (2007).

[51] M. Zwierzycki, P. A. Khomyakov, A. A. Starikov, K. Xia, M. Talanana, P. X. Xu, V. M. Karpan, I. Marushchenko, I. Turek, G. E. W. Bauer, G. Brocks, and P. J. Kelly, Calculating scattering matrices by wave function matching, Phys. Stat. Sol. B 245, 623 (2008).

[52] Tatiane P. Santos, Leandro R. F. Lima, and Caio H. Lewenkopf, An order $N$ numerical method to efficiently calculate the transport properties of large systems: An 
algorithm optimized for sparse linear solvers, J. Comput. Phys. 394, 440 (2019).

[53] Pengke Li and Ian Appelbaum, Electrons and holes in phosphorene, Phys. Rev. B 90, 115439 (2014).

[54] A. N. Rudenko and M. I. Katsnelson, Quasiparticle band structure and tight-binding model for single- and bilayer black phosphorus, Phys. Rev. B 89, 201408(R) (2014).

[55] See the Supplemental Material at http://link.aps.org/supple mental/10.1103/PhysRevApplied.12.064025 for a detailed discussion of the classification of the crystal directions.

[56] Yea-Lee Lee, Fangzhou Zhao, Ting Cao, Jisoon Ihm, and Steven G. Louie, Topological phases in cove-edged and chevron graphene nanoribbons: Geometric structures, $Z_{2}$ invariants, and junction states, Nano Lett. 18, 7247 (2018).

[57] Z. F. Wang, Qunxiang Li, Q. W. Shi, Xiaoping Wang, J. G. Hou, Huaixiu Zheng, and Jie Chen, Ballistic rectification in a Z-shaped graphene nanoribbon junction, Appl. Phys. Lett. 92, 133119 (2008).

[58] Rui Zhang, X. Y. Zhou, D. Zhang, W. K. Lou, F. Zhai, and Kai Chang, Electronic and magneto-optical proper- ties of monolayer phosphorene quantum dots, 2D Mater. 2 , 045012 (2015).

[59] Rui Zhang, Zhenhua Wu, X. J. Li, and Kai Chang, Aharonov-Bohm effect in monolayer phosphorene nanorings, Phys. Rev. B 95, 125418 (2017).

[60] Enrique Montes and Udo Schwingenschlögl, Highperformance field-effect transistors based on $\alpha \mathrm{P}$ and $\beta \mathrm{P}$, Adv. Mater. 31, 1807810 (2019).

[61] Katsunori Wakabayashi and Manfred Sigrist, ZeroConductance Resonances due to Flux States in Nanographite Ribbon Junctions, Phys. Rev. Lett. 84, 3390 (2000).

[62] Anffany Chen, R. Ilan, F. de Juan, D. I. Pikulin, and M. Franz, Quantum Holography in a Graphene Flake with an Irregular Boundary, Phys. Rev. Lett. 121, 036403 (2018).

[63] S. Datta, Electronic Transport in Mesoscopic Systems (Cambridge University Press, Cambridge, 1995).

[64] Motohiko Ezawa, Minimal models for Wannier-type higher-order topological insulators and phosphorene, Phys. Rev. B 98, 045125 (2018). 